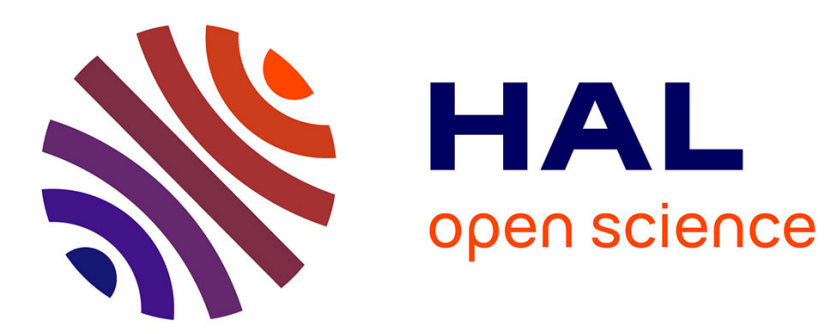

\title{
Production de diacétyle dans les laits et les crèmes soumis à la neutralisation au cours de la fermentation lactique
}

\author{
R. Lacrosse
}

\section{- To cite this version:}

R. Lacrosse. Production de diacétyle dans les laits et les crèmes soumis à la neutralisation au cours de la fermentation lactique. Le Lait, 1969, 49 (487), pp.434-438. hal-00928496

\section{HAL Id: hal-00928496 https://hal.science/hal-00928496}

Submitted on 1 Jan 1969

HAL is a multi-disciplinary open access archive for the deposit and dissemination of scientific research documents, whether they are published or not. The documents may come from teaching and research institutions in France or abroad, or from public or private research centers.
L'archive ouverte pluridisciplinaire HAL, est destinée au dépôt et à la diffusion de documents scientifiques de niveau recherche, publiés ou non, émanant des établissements d'enseignement et de recherche français ou étrangers, des laboratoires publics ou privés. 


\title{
Production de diacétyle dans les laits et les crèmes soumis à la neutralisation au cours de la fermentation lactique
}

\author{
par \\ par R. LACROSSE, \\ Chef de travaux - Station laitière de l'Etat de Gembloux (Belgique)
}

\section{I. - INTRODUCTION}

Plusieurs chercheurs ont constaté que les beurres produits dans les usines laitières et en particulier les beurres fabriqués à partir de crèmes fraîches ont tendance lorsque le $\mathrm{pH}$ atteint en fin de maturation est bas, à prendre un goût oxydé et graisseux (1).

On peut situer la valeur du $\mathrm{pH}$ limite aux environs de 5,0 et afin d'éviter l'apparition de ce défaut, les beurreries ont été amenées à modifier la conduite de la maturation des crèmes en abaissant notamment la température de fermentation.

La production de beurre moins acide entraîne une diminution de la teneur en diacétyle et on arrive ainsi à obtenir des beurres moins aromatiques, de goût neutre, assez plat. Les produits aromatiques caractéristiques du beurre et en particulier le diacétyle accusent une teneur maximale dans la zone de fermentation dont le pH est compris entre 5,0 et 4,6 (2). Nos essais ont eu pour but de déterminer l'influence de la neutralisation à un $\mathrm{pH}$ fixé sur la production de diacétyle dans les laits et les crèmes soumis à la fermentation lactique.

\section{II. - CONDITIONS DES ESSAIS}

Les expériences ont été réalisées en étuve thermostatique, la neutralisation effectuée par un pH-mètre régulateur Radiometer Modèle TTT1. Les laits et les crèmes étaient soumis à la maturation pendant $\pm 17 \mathrm{~h}$ et la neutralisation effectuée par une solution de $\mathrm{NaOH} 5 \mathrm{~N}$. Chaque essai comportait un échantillon témoin non neutralisé, la comparaison étant rendue possible entre les deux modes de fermentation. 
Afin de répartir la soude servant à la neutralisation uniformément dans la masse du produit testé, nous avons dû procéder à une certaine agitation pendant la fermentation.

\section{a) Expériences sur laits}

Température d'incubation $22^{\circ} \mathrm{C}$.

Ensemencement à 1 p. 100 dans du lait stérilisé homogénéisé.

Le $\mathrm{pH}$ était maintenu constant aux environs de 5,2.

TABLEAU 1

Résultats enregistrés

\begin{tabular}{|c|c|c|c|}
\hline \multicolumn{2}{|c|}{ Essais témoins } & \multicolumn{2}{|c|}{ Essais avec neutralisation } \\
\hline $\mathrm{pH}$ & Diacétyle $\mathrm{mgr} / \mathrm{kg}$ & $\mathrm{pH}$ & Diacétyle $\mathrm{mgr} / \mathrm{kg}$ \\
\hline \multirow[t]{2}{*}{$\begin{array}{l}4,60 \\
4,55 \\
4,65 \\
4,50 \\
4,40 \\
4,45 \\
4,60\end{array}$} & $\begin{array}{l}3,55 \\
1,52 \\
4,84 \\
4,08 \\
2,50 \\
1,32 \\
2,73\end{array}$ & $\begin{array}{l}5,20 \\
5,25 \\
5,20 \\
5,28 \\
5,20 \\
5,23 \\
5,25\end{array}$ & $\begin{array}{l}3,77 \\
1,55 \\
4,08 \\
3,44 \\
4,50 \\
5,50 \\
6,50\end{array}$ \\
\hline & Mo $=2,94$ & & Mo $=4,19$ \\
\hline
\end{tabular}

Les résultats se sont donc révélés dans l'ensemble assez encourageants car nous constatons une teneur moyenne en diacétyle sensiblement plus élevée dans les échantillons neutralisés que dans les échantillons témoins qui correspondent à la méthode généralement employée pour la fabrication des levains en beurrerie.

\section{b) Expériences sur crèmes}

Les échantillons devant être soumis à une agitation constante en cours de fermentation, nous avons rencontré des difficultés pour la conduite des essais car un certain barattage se produisait en fin de fermentation.

Nous avons obvié à cet inconvénient en diluant la crème par addition d'une quantité d'environ $1 / 3$ de leur volume d'eau stérile, nous obtenions de cette manière une crème contenant \pm 25 p. 100 de matière grasse butyrique.

Conditions de l'essai

Pasteurisation de la crème à $85^{\circ} \mathrm{C}$ pendant $5 \mathrm{mn}$.

Température de fermentation : $15^{\circ} \mathrm{C}$.

Quantité de ferment ajoutée : 7 p. 100. 
Neutralisation avec solution $\mathrm{NaOH} 5 \mathrm{~N}$.

Durée de l'acidification : $18 \mathrm{~h}$.'

TABLEAU 2

\begin{tabular}{|c|c|c|c|c|}
\hline \multirow{2}{*}{$\begin{array}{l}\mathrm{pH} \text { initial de la crème } \\
\text { (avec addition du } \\
\text { ferment) }\end{array}$} & \multicolumn{2}{|c|}{ Crème témoin } & \multicolumn{2}{|c|}{ Crème neutralisée } \\
\hline & $\begin{array}{c}\mathrm{pH} \\
\text { final }\end{array}$ & $\begin{array}{l}\text { Diacétyle } \\
\text { en } \mathrm{mg} / \mathrm{kg}\end{array}$ & $\begin{array}{l}\mathrm{pH} \\
\text { final }\end{array}$ & $\begin{array}{c}\text { Diacétyle } \\
\mathrm{mg} / \mathrm{kg}\end{array}$ \\
\hline $\begin{array}{l}6,30 \\
5,80 \\
5,75 \\
5,60 \\
5,50 \\
5,70\end{array}$ & $\begin{array}{l}4,60 \\
4,70 \\
4,60 \\
4,70 \\
4,80 \\
4,75\end{array}$ & $\begin{array}{l}2,30 \\
2,50 \\
2,58 \\
1,03 \\
1,85 \\
1,90\end{array}$ & $\begin{array}{l}5,25 \\
5,20 \\
5,10 \\
5,30 \\
5,20 \\
5,25\end{array}$ & $\begin{array}{l}2,07 \\
2,41 \\
3,72 \\
2,33 \\
2,86 \\
3,35\end{array}$ \\
\hline & & $\mathrm{Mo}=2,02$ & & Mo $=2,79$ \\
\hline
\end{tabular}

Une autre série d'essais a été effectuée au moyen de crèmes non diluées à teneur en matière grasse plus faible afin d'éviter le barattage en cours de maturation.

Conditions de l'essai

Pasteurisation de la crème à $85^{\circ} \mathrm{C}$ pendant $5 \mathrm{mn}$.

Température de fermentation : $17^{\circ} \mathrm{C}$.

Quantité de ferment ajoutée : 5 p. 100.

Neutralisation par $\mathrm{NaOH} 5 \mathrm{~N}$.

Durée de l'acidification : $18 \mathrm{~h}$.

TABLEAU 3

\begin{tabular}{|c|c|c|c|c|}
\hline \multirow{2}{*}{$\begin{array}{l}\mathrm{pH} \text { initial de la crème } \\
\text { (avec addition du } \\
\text { ferment) }\end{array}$} & \multicolumn{2}{|c|}{ Crème témoin } & \multicolumn{2}{|c|}{ Crème neutralisée } \\
\hline & $\mathrm{pH}$ & $\begin{array}{c}\text { Diacétyle } \\
\mathrm{mg} / \mathrm{kg}\end{array}$ & $\mathrm{pH}$ & $\begin{array}{c}\text { Diacétyle } \\
\mathrm{mg} / \mathrm{kg}\end{array}$ \\
\hline 5,60 & 4,60 & 4,20 & 5,20 & 4,40 \\
\hline 5,80 & 4,75 & 7,00 & 5,20 & 8,50 \\
\hline 5,60 & 4,55 & 4,60 & 5,10 & 7,40 \\
\hline 5,90 & 4,55 & 5,60 & 5,10 & 8,00 \\
\hline 6,00 & 4,60 & 5,50 & 5,15 & 5,50 \\
\hline 6,00 & 4,60 & 5,50 & 5,15 & 4,80 \\
\hline 6,20 & 4,60 & 2,30 & 5,20 & 2,40 \\
\hline 6,30 & 4,60 & 2,15 & 5,15 & 2,15 \\
\hline 6,30 & 4,60 & 5,10 & 5,20 & 2,07 \\
\hline \multirow[t]{2}{*}{6,20} & 4,75 & 7,55 & 5,10 & 9,20 \\
\hline & & $\mathrm{Mo}=4,94$ & & $\mathrm{Mo}=5,44$ \\
\hline
\end{tabular}




\section{DISCUSSION ET CONCLUSIONS}

Les résultats enregistrés lors de la fermentation des crèmes sont sensiblement les mêmes dans les crèmes dont l'acidification est fortement accentuée $(\mathrm{pH} \pm 4,60)$ que dans les crèmes soumises à neutralisation $(\mathrm{pH} \pm 5,2)$.

Dans la phase qui suit le commencement de la neutralisation, le nombre de bactéries lactiques continue à s'accroître fortement et en même temps que la production d'acide lactique neutralisé au fur et à mesure de sa production on assiste à l'apparition progressive des produits accessoires de fermentation et en particulier du diacétyle.

Accolas et Auclair (3) préconisent cette technique de neutralisation automatique afin d'obtenir des cultures à très grande teneur en cellules bactériennes, ils ont obtenu en milieu de culture un nombre de bactéries fortement supérieur à celui dénombré en cultivant sans neutralisation.

Nous ne pouvons préjuger des applications pratiques qui pourraient entraîner les résultats que nous avons enregistrés au cours de nos essais de neutralisation automatique. Il semble que cette technique pourrait donner lieu à certaines applications intéressantes dans le domaine de la production de levains lactiques à haute teneur en diacétyle. Dans le domaine de la maturation des crèmes en beurrerie, elle pourrait se révéler utile mais il serait toutefois nécessaire d'envisager également les conséquences de l'application d'une telle technique sur les propriétés organoleptiques du produit fini, le beurre.

\section{Rés u m é}

L'auteur donne les résultats d'essais sur la neutralisation automatique de levains lactiques à $\mathrm{pH} 5,0-5,2$, des essais ont été effectués également sur des crèmes mises en maturation. Il a pu constater une augmentation sensible du diacétyle dans les levains soumis à neutralisation par rapport aux levains acidifiés dans les conditions normales. Les résultats étaient moins favorables dans les crèmes soumises à la maturation.

\section{S u m m a ry}

The author gives the results of experiments on the automatic

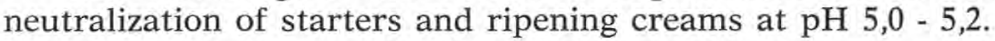

He remarks a sensible increasing of biacetyl in the neutralizing starters in comparison with acidified starters in normal conditions.

The results where not so suitable with ripening creams. 


\section{Bibliographie}

[1] Piraux (E.). - La qualité du beurre, ses composants et les moyens de l'apprécier. Compte rendu de recherches IRSIA, 34, juillet 1966, p. 81.

[2] Même p. 96.

[3] Accolas (J. P.), Auclair (J.). - Conservation de l'état congelé de suspension de bactéries lactiques concentrées sous faible volume. Le Lait, 465-466, 1967, p. 264.

Reçu pour publication en février 1969. 